\title{
Uso de derivados da cannabis sativa. $L$ no tratamento adjuvante em pacientes oncológicos no município de Caruaru, Pernambuco
}

\author{
Use of cannabis sativa. $L$ derivatives. in adjuvant treatment in oncological patients in the city of \\ Caruaru, Pernambuco \\ Uso de derivados de cannabis sativa. $L$ en tratamiento adyuvante en pacientes oncológicos de la \\ ciudad de Caruaru, Pernambuco
}

\section{Resumo}

No contexto atual o uso medicinal de derivados da cannabis tem tomado proporções crescentes. Entretanto, embora o uso medicinal tenha obtido nos últimos anos um aumento significativo e aprovação judicial para o uso de forma legal no Brasil o conhecimento e utilização dessa opção terapêutica ainda não é uma realidade. Sendo um dos maiores entraves a burocracia para o acesso aos medicamentos ou produtos. Tendo em vista o cenário de ascensão terapêutica dos medicamentos e produtos derivados da Cannabis Sativa no Brasil e no mundo, esse artigo objetivou a realização de um levantamento através de um estudo de campo quantitativo-descritivo nos centros oncológicos do município de Caruaru-PE para averiguar o posicionamento dos médicos e da equipe multidisciplinar em relação aos produtos em questão para ajudar no tratamento ao câncer; qual o conhecimento dos mesmos sobre essa opção terapêutica bem como fazer um paralelo com as drogas já existentes e a burocracia para ter acesso aos derivados da Cannabis Sativa. Mediante aos resultados o uso da droga em questão apontou para uma neutralidade dos profissionais envolvidos na área oncológica.

Palavras-chave: Derivados da Cannabis Sativa; Câncer; Opção terapêutica.

\begin{abstract}
In the current context, the medicinal use of cannabis derivatives has taken on increasing proportions. However, although medicinal use has obtained a significant increase in recent years and legal approval for its use legally in Brazil, knowledge and use of this therapeutic option is still not a reality. One of the biggest obstacles is the bureaucracy for accessing medicines or products. In view of the therapeutic rise of Cannabis Sativa drugs and products in Brazil and worldwide, this article aimed to carry out a survey through a quantitative-descriptive field study in cancer centers in the city of Caruaru-PE to investigate the positioning of physicians and the multidisciplinary team in relation to the products in question to help with cancer treatment; what is their knowledge about this therapeutic option, as well as making a parallel with the existing drugs and the bureaucracy to have access to Cannabis Sativa derivatives. Based on the results, the use of the drug in question pointed to a neutrality of professionals involved in the oncology area.
\end{abstract}

Keywords: Cannabis Sativa Derivatives; Cancer; Therapeutic option.

\section{Resumen}

En el contexto actual, el uso medicinal de los derivados del cannabis ha adquirido proporciones crecientes. Sin embargo, aunque el uso medicinal ha obtenido un aumento significativo en los últimos años y la aprobación legal para su uso legalmente en Brasil, el conocimiento y uso de esta opción terapéutica aún no es una realidad. Uno de los mayores obstáculos es la burocracia para acceder a medicamentos o productos. Dado el auge terapéutico de los medicamentos y productos de Cannabis Sativa en Brasil y en todo el mundo, este artículo tuvo como objetivo realizar una encuesta a 
través de un estudio de campo descriptivo-cuantitativo en centros oncológicos de la ciudad de Caruaru-PE para investigar la posición de los médicos y el equipo multidisciplinario en relación con los productos en cuestión para ayudar en el tratamiento del cáncer; cuál es tu conocimiento de esta opción terapéutica, además de hacer un paralelismo con las drogas existentes y la burocracia para tener acceso a derivados de Cannabis Sativa. Con base en los resultados, el uso del fármaco en cuestión apuntó a una neutralidad de los profesionales de la oncología.

Palabras clave: Derivados de Cannabis Sativa; Cáncer; Opción terapéutica.

\section{Introdução}

Cannabis é gênero de uma planta, popularmente conhecida no Brasil como maconha, originária da Ásia e pertencente à família Cannabaceae, cujas subespécies mais notórias são a Cannabis sativa ${ }^{1}$ subespécie sativa, a Cannabis sativa subespécie indica e a Cannabis sativa subespécie ruderalis. Elas se diferenciam, principalmente, em função do modo de crescimento, quantidade de princípios ativos e características morfológicas. Devido ao clima temperado e tropical, a espécie predominante em solo brasileiro é a Cannabis sativa sativa (Gurgel et al 2019).

Cannabis Sativa L é uma planta que apresenta grande potencial terapêutico, apesar de suas propriedades psicotrópicas. Esta planta vem sendo utilizada, há séculos, pela humanidade para diversos fins, tais como, alimentação, rituais religiosos e práticas medicinais (Mechoulam. R apud Ribeiro, 2014).

A grande potencialidade medicinal da Cannabis deve-se ao grande número de substâncias químicas, os chamados canabinoides descobertos na planta, que chegam a mais de 400 componentes. E, apesar de ter sido bastante utilizada na antiguidade para diversas finalidades, seus efeitos colaterais adversos fizeram com que fosse vista negativamente, levando à proibição e marginalização em muitos países, inclusive no Brasil. Isso aconteceu por diversos motivos relacionados ao fato de que, na época, os princípios ativos da planta ainda não haviam sido isolados e estudados, e os extratos geravam efeitos inconsistentes e diversos, sendo muitas vezes indesejáveis (Carneiro, 2018).

A primeira utilização da planta de forma documentada foi por volta de 2300 a.C., pelo chinês Chen Nong, classificada por ele como um dos "supremos elixires da imortalidade" era utilizada no tratamento de muitas doenças como constipação intestinal, dores, malária, expectoração, epilepsia, tuberculose, depressão, nos sintomas psiquiátricos como: hipnótico e tranquilizante no tratamento de ansiedade, mania e histeria (Cebulski, F.S \& Martins, C.A.F, 2016).

Documentos históricos mostram que a maconha foi introduzida no Brasil na época das capitanias, também com a utilização de suas fibras, estimava-se que a planta já fosse conhecida há mais tempo, pelos primeiros escravos, que a utilizavam como hipnótico, mostrando assim que a droga apresentava propriedades terapêuticas (Moreau, 2008 APUD Santos, 2017).

A ampla distribuição de receptores canabinoides em nosso corpo, principalmente no SNC, sistema imunológico e terminais nervosos, explica a amplitude dos possíveis efeitos terapêuticos. Pesquisas relacionadas aos canabinoides revelaram a importância do sistema endocanábico, que não só controla a transmissão dos estímulos dolorosos em vários níveis, mas também controla o sistema opioide, também envolvido no controle da dor (Exposito, 2013).

Os receptores canabinóides CB1 e CB2 são diferenciados pela forma como transmitem o sinal e por sua distribuição em diferentes tecidos. A ativação dos receptores canabinóides resulta na inibição da adenilatociclase, que impede a conversão de ATP em AMP cíclico; por outro lado, sua interação com alguns canais iônicos. Ambos CB1 e CB2 pertencem à ampla família de receptores acoplados à proteína $\mathrm{G}$. A ativação do receptor $\mathrm{CB} 1$ resulta em efeitos no processamento da memória, humor, sono, regulação motora, apetite e sensação de dor, enquanto a ativação CB2 não produz esses efeitos. CB1s são encontrados principalmente no córtex, medula espinhal e neurônios do sistema nervoso periférico, embora também estejam presentes em

\footnotetext{
${ }^{1}$ Cannabis Sativa, também conhecida por vários nomes populares (Maconha, erva, marijuana, cânhamo, haxixe, bagha, entre outros) é uma planta da família das Canabiáceas, Biologicamente, a Cannabis faz parte do gênero de plantas angiospermas que produzem flor, cultivada em várias regiões de todo o mundo, a Cannabis atualmente refere-se a drogas psicoativas e medicamentos derivados da planta (Carneiro, 2018).
} 
certos órgãos e tecidos periféricos, como glândulas endócrinas, glândulas salivares, leucócitos, baço, coração e em certas áreas do aparelho reprodutor, sistemas urinário e gastrointestinal (Sánchez, 2019).

Atualmente os derivados da cannabis sativa para fins medicinais são legais em um leque de países como é o caso dos Estados Unidos, Canadá, Israel, Holanda e Uruguai. No Brasil o uso é regularizado mediante a RDC 327, de 09 de dezembro de 2019. "dispõe sobre os procedimentos para a concessão da Autoriza Sanitária para a fabricação e a importação, bem como estabelece requisitos para a comercialização, prescrição, a dispensação, o monitoramento e a fiscalização de produtos de Cannabis para fins medicinais, e dá outras providências". Com isso, a proposta deste trabalho fora investigar a questão da acessão do uso dos produtos derivados da Cannabis no município de Caruaru-PE nos centros de tratamentos oncológicos.

\section{Metodologia}

Para Fonseca (2002) a pesquisa é uma atividade nuclear da ciência. Ela possibilita uma aproximação e um entendimento da realidade a investigar. A pesquisa é um processo permanentemente inacabado. Processa-se através de aproximações sucessivas da realidade, fornecendo-nos subsídios para uma intervenção no real.

O pressente trabalho trata-se de uma pesquisa de campo quantitativa-descritiva, ainda para Fonseca (2002) os resultados da pesquisa quantitativa podem ser quantificados. Como as amostras geralmente são grandes e consideradas representativas da população, os resultados são tomados como se constituíssem um retrato real de toda a população alvo da pesquisa. Com isso, a pesquisa foi realizada nos centros e hospitais que prestam serviços oncológicos no município de Caruaru-PE, com aplicação de questionários específicos. A pesquisa fora realizada nos próprios consultórios dos médicos oncologistas convidados para responderem o questionário. A amostra da pesquisa concerne a médicos e profissionais da saúde que atuam na área da oncologia no município de Caruaru-PE.

O critério de inclusão deu-se a partir da filtragem da área de atuação clínica dos profissionais. De modo que apenas médicos oncologistas e integrantes da equipe multidisciplinar que trabalham em prol do diagnóstico e do tratamento em indivíduos com câncer participaram da pesquisa. Portanto, os profissionais que não correspondem a área clínica da oncologia foram excluídos da pesquisa. A coleta de dados deu-se por meio de uma pesquisa com survey que de acordo com o Babbie (1999) é particularmente semelhante ao tipo de pesquisa de "censo", onde o que diferencia as duas pesquisas é que o "survey examina uma amostra da população, enquanto o censo geralmente implica uma enumeração da população toda. A coleta de dados foi baseada na aplicação de questionário fechado, elaborado com base na metodologia desenvolvida por Schwartz (1992) contendo perguntas específicas relativo à caracterização do público alvo do estudo e do conhecimento sobre Cannabis Sativa. De modo que, os resultados foram analisados através de um banco de dados criado no programa Excel - Windows ${ }^{\circledR}$

O presente estudo foi aprovado pelo Comitê de Ética em Pesquisa do Centro Universitário do Vale do Ipojuca sob o parecer de número 4.920.923. Aos sujeitos participantes da pesquisa tiveram garantido o respeito aos princípios de autonomia, não maleficência e confidencialidade das informações obtidas, respeitando assim as diretrizes e critérios estabelecidos na Resolução 466/12 do Conselho Nacional de Saúde (CNS) que tem como base considerar o respeito pela dignidade humana e pela especial proteção devida aos participantes das pesquisas científicas envolvendo seres humanos. Todas essas informações estavam presentes no Termo de consentimento livre e esclarecido (TCLE).

\section{Resultados}

O estudo foi realizado no município de Caruaru- PE que fica situada na região nordeste do Brasil. 10 médicos, 1 farmacêutico e 5 profissionais que compõem a equipe técnica voltada ao acompanhamento de pacientes oncológicos foram convidados a fazer parte da pesquisa. No entanto, apenas 5 participantes aceitaram fazer parte da pesquisa ( $60 \%$ Médicos; $\mathrm{n}=3$ ) 
(40 \% Enfermeiros; n=2) (Gráfico 1). Não houve perda amostral de nenhum participante por preenchimento errôneo dos questionários. A média de idade dos participantes foi de 38 anos. O estudo ocorrera entre o mês de agosto e se findou em meados do mês de outubro de 2021, de modo que os profissionais responderam um questionário de forma anônima.

Gráfico 1 - Número de participantes em porcentagem.

\section{Participantes}

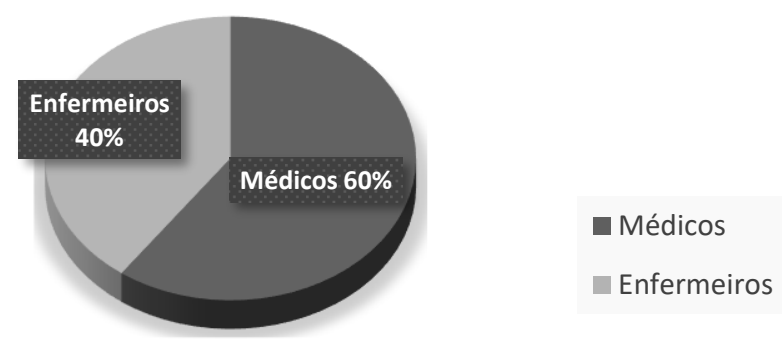

Fonte: Autores (2021).

Embora a amostragem da pesquisa tenha sido pequena, se foi possível constatar a carência de profissionais que já trabalham prescrevendo medicamentos/produtos derivados da Cannabis Sativa no município de Caruaru-PE. Os médicos que foram entrevistados alegaram ter conhecimento sobre os medicamentos/produtos (Tabela 1). No entanto, nunca fizeram prescrição, mesmo não havendo preconceito com o medicamento/produto em questão). Uma vez que, os enfermeiros além de nunca terem acompanhado pacientes que faziam uso dos derivados da Cannabis Sativa não tinham conhecimento sobre os derivados em questão (Tabela 2).

Tabela 1 - Conhecimento dos médicos oncologistas sobre os derivados da Cannabis Sativa $(\mathrm{n}=3)$.

\section{Conhecimento sobre medicamentos e produtos \\ derivados da Cannabis Sativa para ajudar em efeitos colaterais da quimioterapia:}

\begin{tabular}{lcc} 
& $\mathbf{N}(\boldsymbol{\%})$ & $\mathbf{1 0 0 \%}$ \\
\hline Sim & $\mathbf{0 0}$ & \\
\hline Não & & 0 \\
\hline
\end{tabular}

Fonte: Dados coletados pelos autores no município de Caruaru, Pernambuco, 2021.

Tabela 2 - Conhecimento dos enfermeiros oncologistas sobre os derivados da Cannabis Sativa $(\mathrm{n}=2)$.

\section{Conhecimento sobre medicamentos e produtos derivados da Cannabis Sativa para ajudar em efeitos colaterais da quimioterapia:}

\begin{tabular}{lll} 
& $\mathbf{N}(\boldsymbol{\%})$ \\
Sim & $\mathbf{0 \%}$ & $\mathbf{1 0 0 \%}$ \\
\hline Não & $\mathbf{0 0}$ \\
\hline
\end{tabular}

Fonte: Dados coletados pelos autores no município de Caruaru, Pernambuco (2021).

\section{Discussão}

\section{Câncer}

Câncer é um termo que abrange mais de 100 diferentes tipos de doenças malignas que têm em comum o crescimento desordenado de células, que podem invadir tecidos adjacentes ou órgãos a distância. Dividindo-se rapidamente, estas células tendem a ser muito agressivas e incontroláveis, determinando a formação de tumores, que podem espalhar-se para outras regiões do corpo. Os diferentes tipos de câncer correspondem aos vários tipos de células do corpo. Quando começam em tecidos 
epiteliais, como pele ou mucosas, são denominados carcinomas. Se o ponto de partida são os tecidos conjuntivos, como osso, músculo ou cartilagem, são chamados sarcomas (Inca, 2020).

O câncer, nos dias atuais, tornou-se um problema de saúde pública mundial, uma vez que tem aumentado sua prevalência dentro das doenças crônicas não transmissíveis, necessitando de grandes investimentos financeiros e acarretando ônus institucional e social para os países (Sawada, et al, 2009). De acordo com os dados do Instituto Nacional de Câncer (ICIA) Para o Brasil, a estimativa para cada ano do triênio 2020-2022 aponta que ocorrerão 625 mil casos novos de câncer (450 mil, excluindo os casos de câncer de pele não melanoma). O câncer de pele não melanoma será o mais incidente (177 mil), seguido pelos cânceres de mama e próstata (66 mil cada), cólon e reto (41 mil), pulmão (30 mil) e estômago (21 mil).

\section{Quimioterapia}

Os efeitos adversos da quimioterapia ${ }^{2}$ podem ser divididos em dois grupos: agudos, que se iniciam minutos após a a administração dos agentes antineoplásicos e persistem por alguns dias, e tardios, que aparecem semanas ou meses após a infusão dos mesmos (Schlosser; Ceolim, 2012).

Para Valentin et al (2021) A caquexia é uma síndrome associada ao câncer avançado, HIV, pacientes em quimioterapia e aqueles que são acompanhados em cuidados paliativos. A prevalência é de $25 \%$ dos pacientes com diagnóstico de câncer, $26 \%$ dos que recebem quimioterapia e 14 a $38 \%$ dos pacientes com HIV. A base do controle é a cannabis, devido ao efeito do delta9-tetra-hidrocanabinol (THC), do qual o dronabinol, uma droga desenvolvida para estimular o apetite e o ganho de peso, foi derivado. Os receptores CB1 possuem alta densidade no sistema nervoso central, sendo localizados em hipocampo, bulbo e cerebelo. Já o receptor CB2, apesar de ser expresso também no sistema nervoso central, tem alta densidade localizada no sistema imunológico como representado na (figura 1) (Amaral, 2020).

Figura 1. Localização dos receptores canabinoides CB1 e CB2 no corpo humano

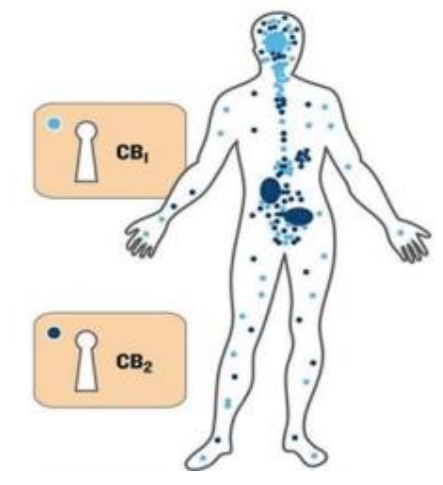

Fonte: Amaral (2020).

De acordo com um estudo realizado por Amato et al (2017) a Nabilona mostrou-se significativamente superior à Proclorperazina, Domperidona e Alizaprida por tratamento de náuseas e vômitos associados à quimioterapia para câncer quando usado de forma contínua.

Em usos clínicos limitados, os canabinoides atuam como antieméticos contra a fase aguda e tardia de vômitos induzidos por quimioterapia; além disso, o efeito antiemético dos canabinóides exógenos parece melhorar quando combinados com os antieméticos convencionais, como metoclopramida, lorazepam, entre outros (Netzahualcoyotzi-stone et al).

\footnotetext{
${ }^{2}$ A quimioterapia é reconhecida pelos efeitos colaterais que acometem os pacientes durante o tratamento, principalmente náuseas, vômitos, alopecia, diarreia ou constipação e outros mais, que comprometem a qualidade de vida das pessoas de forma global Associadas a isso, há alterações na aparência física, dificuldade para manter um vínculo empregatício e as relações interpessoais (Wakiuchi et al, 2019).
} 
A (Tabela 3) a seguir fornece um resumo dos medicamentos abordados anteriormente que são derivados da Cannabis Sativa utilizados para o tratamento de doenças como o câncer, esclerose múltipla, AIDS.

Tabela 3 - Medicamentos derivados da cannabis sativa

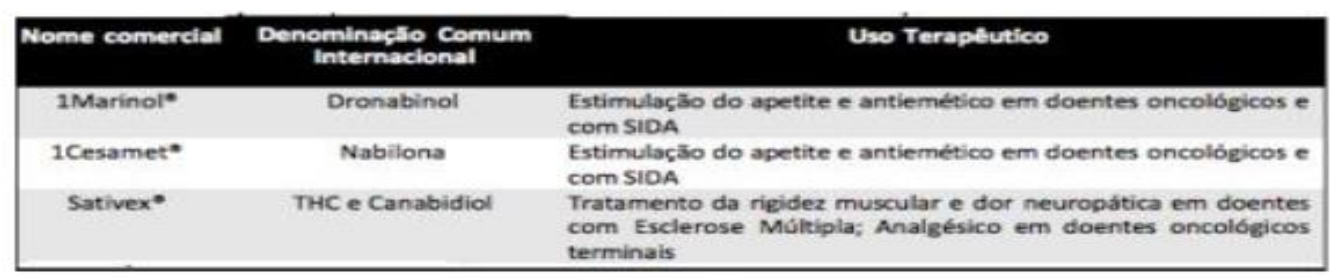

Fonte: Sunaga (2018).

Em estudos cruzados em que os pacientes receberam produtos à base de cannabis e antieméticos convencionais, os pacientes preferiram os medicamentos à base de cannabis. Os medicamentos à base de cannabis podem ser úteis no tratamento de náuseas e vômitos induzidos por quimioterapia, que respondem mal aos antieméticos convencionais (Bridgeman, 2017).

Embora a aprovação dos medicamentos derivados da Cannabis Sativa de forma controlada pela a Anvisa tenha ocorrido desde o final do ano de 2019, de acordo com os resultados provenientes do presente estudo o uso desses medicamentos ainda não é uma realidade no município de Caruaru- PE. Os resultados da pesquisa apontaram para uma neutralidade sobre o uso dos derivados da cannabis sativa pelos médicos oncologistas que responderam ao questionário. Tal neutralidade refletiu no conhecimento dos enfermeiros que fazem parte da equipe técnica de suporte aos pacientes oncológicos. O número de profissionais que se recusaram a fazer parte da pesquisa é um forte indicio de preconceito e falta de interesse para com o uso desses derivados. O preço dos medicamentos e a burocracia ainda enfrentada para a obtenção são fatores que podem influenciar no interesse por essa opção terapêutica.

O fato dos médicos oncologistas que participaram da pesquisa terem conhecimentos sobre esses medicamentos é um ponto positivo. De modo que ainda é uma opção medicamentosa muito recente, carecendo então de interesse no quesito de atualização dos profissionais da área oncológica.

Uma possível forma de mudar esse cenário de prescrição e consequentemente utilização desses medicamentos seria a implantação dos mesmos no sistema únicos de saúde (SUS). Ainda no final de 2019, o Ministério da Saúde sinalizou possibilidade de avaliar a incorporação ao SUS de medicamentos à base de Cannabis. No entanto, até o dado momento nenhuma implementação fora feita.

\section{Conclusão}

Embora os medicamentos derivados da Cannabis Sativa já possuam aprovação e indicação para o seu uso adjuvante contra os efeitos colaterais da quimioterapia em pacientes oncológicos, o uso desses medicamentos ainda não é uma realidade para pacientes com câncer no município de Caruaru-Pe. Esse cenário pode se dar ao fato da dificuldade para obtenção da prescrição e consequentemente do medicamento. Os resultados da pesquisa refletiram uma carência de interesse para com os medicamentos em questão, mesmo que os dados apontem para uma ausência de preconceito dos profissionais envolvidos no estudo. $\mathrm{O}$ fato de a amostragem da pesquisa ser pequena aponta para resultados limitados. Uma vez que, os profissionais que aceitaram fazer parte do estudo demonstraram neutralidade mediante a utilização desses medicamentos para ajudar nos efeitos colaterais em pacientes submetidos a quimioterapia. 
Research, Society and Development, v. 10, n. 15, e78101522631, 2021

(CC BY 4.0) | ISSN 2525-3409 | DOI: http://dx.doi.org/10.33448/rsd-v10i15.22631

\section{Referências}

Amaral A et al. (2020). Potenciais terapêuticos dos canabinoides. 9(2),.63-76.

Amato, L. et al (2017). revisão da segurança e eficácia terapêutica da cannabis em pacientes com esclerose múltipla, dor neuropática e em pacientes oncológicos tratados com quimioterapia. 41(5-6):279-293.

Babbie, E. (1999). Métodos de pesquisas de survey. ed. UFMG, 519 p.

Bridgeman, M. P, \& Abazia, D. T. Cannabis medicinal: história, farmacologia e implicações para o ambiente de tratamento agudo.

Brasil. Diário oficial da união. Resolução da diretoria colegiada - RDC No 327, de 9 de dezembro de 2019. edição: 239 | seção: 1 | página: 194, Brasil IN.GOV.BR/EN/WEB/DOU/-I

Carneiro, A. C. (2018). Uso medicinal de cannabis sativa. Anápolis, curso de direito - Unievangélica

Cebulski, F. S., \& Martins, C.A.F. (2016). Uso da Cannabis sativa no Tratamento de Doenças Neoplásicas: Uma Visão Biomédica.

Expósito, C. L. (2003). El Cannabis en la práctica clínica. Revista de la Facultad de Medicina, 26(2), 127-130.

Fonseca, J.J.S (2002). Metodologia da pesquisa cientifica. UEC.

Gurgel, H. 1. C et al. (2019). Uso terapêutico do canabidiol: a demanda judicial no estado de Pernambuco, Brasil. Saúde e Sociedade. 28(3), $283-295$.

Honório, K.M; Arroio, A \& Silva, A.B.F.S. (2006). Aspectos terapêuticos de compostos da planta Cannabis sativa. Quím. Nova. 29(2).

Icia (2020). Instituto Nacional do Câncer. https://www.inca.gov.br/o-que-e-cancer

Inca, Instituto Nacional de Câncer (2011). ABC do câncer: abordagens básicas para o controle do câncer / Instituto Nacional de Câncer. Inca, 128 p.

Netzahualcoyotzi-piedra, C et al. (2009). A maconha e o sistema endocanabinoide: dos efeitos recreativos aos terapêuticos. Rev Biomed; 20(2): 128-153

Sánchez, P. R et al. (2019). Therapeutic applications based on cannabinoids action. Gaceta médica de México, $155(3), 12$.

Santos, Q. A. (2017). Revista Saúde em Foco. Edição nº 9.

Sawada, N. O., et al. (2009). Avaliação da qualidade de vida de pacientes com câncer submetidos à quimioterapia. Rev. Esc. Enferm. USP 43.

Schwartz, S. H. (1992). Universals in the content and structure of values: Theory and empirical tests in 20 countries. In M. Zanna (Ed.), Advances in experimental social psychology (Vol. 25, pp. 1-65). Academic Press

Silva, E. F. Q. (2017). Revisão de métodos para determinação de canabinoides em matrizes biológicas por cromatografia líquida de alta eficiência acoplada ao detector de espectrometria de massas.

Sunaga, B. Y. (2018). Efeitos terapêuticos e tóxicos da Cannabis sativa. Trabalho de Conclusão de Curso (Graduação em Farmácia) - Universidade Federal de São Paulo - Campus Diadema, 2018.

Valentín, V et al. (2021). Dronabinol en manejo de apetito y pérdida de peso en pacientes con cáncer terminal, VIH, cuidados paliativos y vejez. Revisión de la literatura. Revista mexicana de anestesiología, 44(3), 207-214.

Wakiuchi, J. et al. (2019). A quimioterapia sob a ótica da pessoa com câncer: uma análise estrutural. v28. 